\title{
Efficient Utilization of 2D Barcode (QR Code) in Boarding Pass for Managing Luggage at Air Port
}

\author{
Awadhesh Kumar \\ AIET Jaipur \\ India
}

\author{
Manish Choubisa \\ AIET Jaipur \\ India
}

\author{
S S Shekhawat \\ AIET Jaipur \\ India
}

\author{
Manish Dubey \\ AIET Jaipur \\ India
}

\begin{abstract}
Quick Response (QR) Code is very useful for encoding the data in an efficient manner. Here data capacity in 2D barcode is limited according to the various types of data formats used for encoding. The data in image format uses more space. The data capacity can be increased by compressing the data using any of the data compression techniques before encoding. In this paper, we suggest a technique for data compression which in turn helps to increase the data capacity of QR Codes generated for image. The main objective of this paper is to present how QR code can be utilized best in boarding pass for managing luggage at air port. Misplace of luggage at air port is very common; here we are proposing an idea for proper handling of misplaced luggage.
\end{abstract}

Keywords: 2D barcodes, Data Capacity, Data Compression, Lossless Compression, QR Code

\section{INTRODUCTION}

Bar codes have become widely popular because of their reading speed, accuracy, and superior functionality characteristics. Barcodes can be divided as 1D, 2D and 3D. 1D barcodes can express information in horizontal direction only. Also, the data capacity is limited. 2D barcodes can hold data both in horizontal and vertical direction. As a result, the data capacity is 100 times more than the 1D barcode [1]. 3D barcode is usually engraved on a product or applied on a product so that the barcode has depth and thickness.

As bar codes became popular and their convenience universally recognized, the market began to call for codes capable of storing more information, more character types, and that could be printed in a smaller space. However, these improvements also caused problems such as enlarging the bar code area, complicating reading operations, and increasing printing cost. 2D Code emerged in response to these needs and problems [2].

QR Code is a kind of 2-D (two-dimensional) symbology developed by Denso Wave and released in 1994 with the primary aim of being a symbol that is interpreted by scanning equipment [3]. 2D bar codes can act like identifier (like in 1D) but takes less space. 2-D barcode minimizes the use of database; alternatively, it functions as database itself.

QR Code holds a considerably greater volume of information than a $1 \mathrm{D}$ bar code. These can be numeric, alphanumeric or binary data - of which up to 2953 bytes can be stored. Only a part of each QR bar code contains actual data, including error correction information. A large area of the QR code is used for defining the data format and version as well as for positioning, alignment and timing purposes. The smallest square dot or pixel element of a QR code is called a module. QR Codes have an empty area around the graphic. This quiet area is ideally 4 modules wide. Examination certificates can also use the QR Encoding techniques [4].

This paper proposes a method in which data capacity can be increased by first compressing the data and then encoding it. Actual requirement for compression arises when we need to encode image data into QR code. A lossless compression technique is proposed to increase the data capacity. For decoding the data, two steps will be followed: (i) decompressing the data using the techniques which are just the reverse of compression technique used here and (ii) decoding the decompressed data. For this, the reverse technique used for encoding the data can be used.

\section{LITERATURE SURVEY}

QR Codes have already overtaken the conventional 1-D bar codes because of the capacity of data that can be stored by a 2-D barcode( $Q R$ Code) is much greater than that of conventional 1-D bar code. QR Code contains data both in horizontal and vertical directions. This stems in many cases from the fact that a typical 1-D barcode can only hold a maximum of 20 characters, whereas as QR Code can hold up to 7,089 characters [3]. QR Codes are capable of encoding the same amount of data in approximately one tenth the space of a traditional 1-D bar code. A great feature of QR Codes is that they do not need to be scanned from one particular angle, as QR Codes can be read regardless of their positioning. The data can be read successfully even if QR code is tampered while 1-D barcode can't. QR Codes can be easily decoded with a smart phone with appropriate barcode reader software (for example:, Kaywa Reader, QRafter and I-Nigma etc.) [5]. Secure communication can also be established using QR Encoding techniques [6]. 


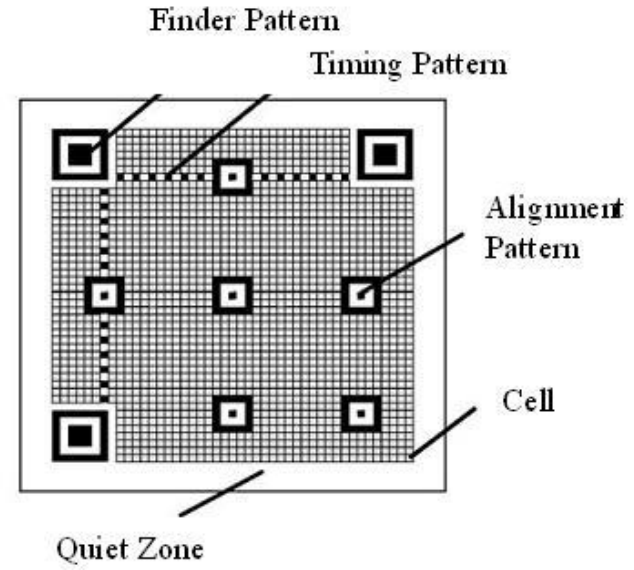

Fig.1: Structure of QR Code

\subsection{Structure of QR Codes}

QR Codes are actually black modules in square patterns on white background but many researchers have been working for colored QR code. It consists of the following areas having specific significance.

- Finder Pattern

- Alignment Pattern

- Timing Pattern

- Quiet Zone

- Data Area

Fig.1 shows the structure of QR Code. The significance of each area is as described as follows:

Each QR Code symbol consists of mainly two regions: an encoding region and function patterns. Function patterns consist of finder, timing and alignment patterns which does not encode any data. The symbol is surrounded on all the four sides by a quiet zone border [7]. A QR Code can be read even if it is tilted or distorted. The size of a QR Code can vary from $21 \times 21$ cells to $177 \times 177$ cells by four cell increments in both horizontal and vertical direction.

\subsubsection{Finder Pattern}

This pattern can be used for detecting the position, size and angle of the QR Code. These can be determined with the help of the three position detection patterns (Finder Patterns) which are arranged at the upper left, upper right and lower left corners of the symbol as shown in Fig. 1.

\subsubsection{Alignment Pattern}

The alignment pattern consists of dark $5 \times 5$ modules, light $3 \times 3$ modules and a single central dark module. This pattern is actually used for correcting the distortion of the symbol [8]. The central coordinate of the alignment pattern will be identified to correct the distortion of the symbol.

\subsubsection{Timing Pattern}

The timing patterns are arranged both in horizontal and vertical directions. These are actually having size similar to one module of the QR Code symbol. This pattern is actually used for identifying the central co-ordinate of each cell with black and white patterns arranged alternately.

\subsubsection{Quiet Zone}

This region is actually free of all the markings. The margin space is necessary for reading the bar code accurately. This zone is mainly meant for keeping the QR Code symbol separated from the external area [9]. This area is usually 4 modules wide.

\subsubsection{Data Area}

It consists of both data and error correction code words. According to the encoding rule, the data will be converted into 0's and 1's. Then these binary numbers will be converted into black and white cells and will be arranged accordingly. Reed-Solomon error correction is also used here [10].

\subsection{Data Capacity}

The data storage capacity of QR Code is very large as compared to 1-D barcode. The number of characters that can be encoded as QR Code varies according to the type of information that is to be encoded. The various information types and the volume that the QR Code can hold are explained in Table 1.

Table 1. Information Types and Volume of Data

\begin{tabular}{|l|c|}
\hline Information Type & Volume of Data \\
\hline Alphabets and Symbols & 4296 \\
\hline Numeric Characters & 7089 \\
\hline Binary Data (8 bit) & 2953 \\
\hline Kanji Characters & 1817 \\
\hline
\end{tabular}

\subsection{Data Compression}

In the history of computer science, data compression, source coding [1] or bit-rate reduction includes encoding information using fewer bits than the original representation. There are two kinds of data compression: lossy and lossless. Lossy compression reduces bits by identifying marginally important information and removing it. Lossless compression reduces bits by identifying and eliminating statistical redundancy. No information is lost in lossless compression.

Data Compression is very useful due to reducing the consumption of resources such as data space or transmission capacity. Because compressed data must be decompressed to be used, this extra processing imposes computational or other costs through decompression. The design of data compression schemes involve trade-offs among various factors, including the degree of compression, the amount of distortion introduced and the computational resources required to compress and uncompress the data [11].

Lossless data compression algorithms usually exploit statistical redundancy to represent data more concisely without losing information. Lossless compression is possible 
because most real-world data has statistical redundancy. The Lempel-Ziv (LZ) compression methods are among the most popular algorithms for lossless compression. DEFLATE is a variation on $\mathrm{LZ}$ which is optimized for decompression speed and compression ratio, but compression can be slow.

\section{PROPOSED TECHNIQUE}

As discussed in [13], the efficiency of QR Codes is increased by applying compression before encoding. This paper focuses the best use of the high capacity QR Code generated in [13]. Till now passengers have to get boarding pass for luggage at air port only. Such boarding pass is using $1 \mathrm{D}$ barcodes for every item. Here the main problems associated with $1 \mathrm{D}$ barcode are: i) needs the access of database every time for retrieving complete information and ii) $1 \mathrm{D}$ barcode get tampered easily. Our approach uses QR code which contains the encrypted information. The advantage of encryption is to secure the information stored on QR code. So that only air port authority can encode the data for proper verification of misplaced luggage. The whole process is described by the following steps:

1. The passenger has to fill his/her journey, personal and luggage details as on ticket online. $\mathrm{He} / \mathrm{she}$ must have the same ID Proof with him/her that is required for authentication of the passenger at the airport.

2. The online system generates a boarding pass having secure barcode for the passenger, which contains encrypted journey and personal details of the passenger. The passenger can take print of secure QR code separately for every item.

3. At the time of check-in at the airport, passenger has to show the QR code on the boarding pass along with ticket to the scanning machine for the verification.

4. Now, the passenger has checked-in the airport successfully.

5. In case of misplace of the luggage during checkout from the air port, the system verifies the luggage left/misplaced with the help of secure QR code affix on it. This verification ensures the authentication of luggage. After successful verification the luggage is handed over to the right passenger. This process ensures the proper handling of misplaced luggage.

The above process is represented in Fig-2(a) and Fig-2(b).

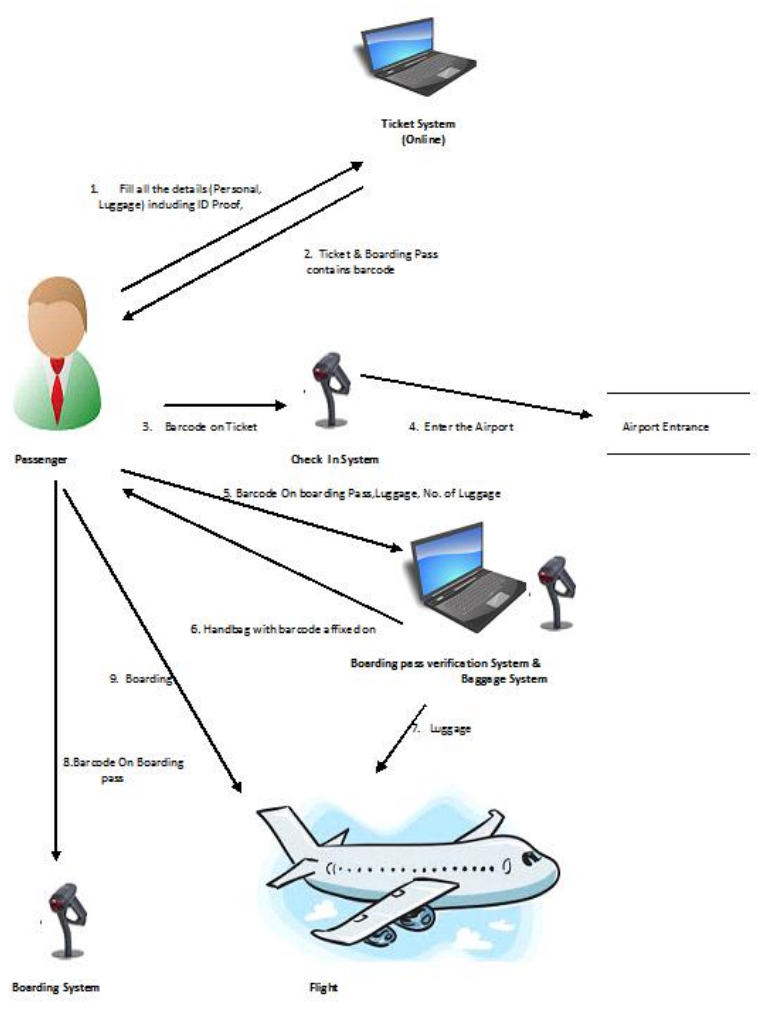

Fig-2(a): Check-in process at Air Port
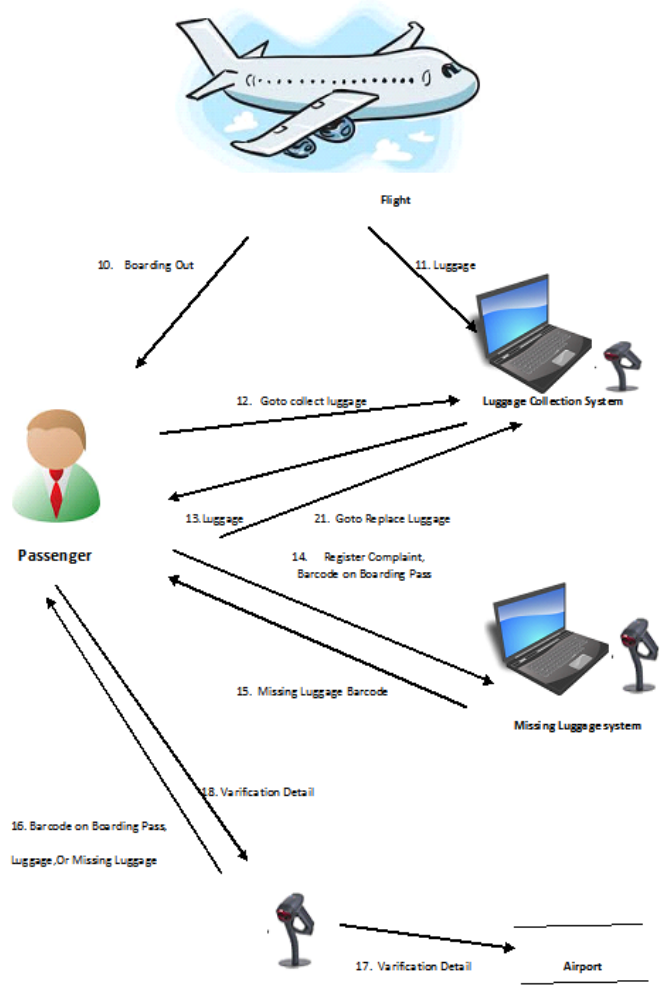

Fig-2(b): Check-in process at Air Port 


\section{RESULTS}

Using the approach discussed above, we are able to provide the facility of generating boarding pass online from home or office. The QR code is generated for every item carried by passenger. He/she can affix QR code on items and the same QR code is on boarding pass also. During check-in at the air port luggage are verified with help of QR code on boarding pass. The misplaced luggage are handled using QR code on it. The whole process is implemented by designing a small web application using C\#.Net on Visual Studio 2008. Fig-3 shows the registration process of luggage:

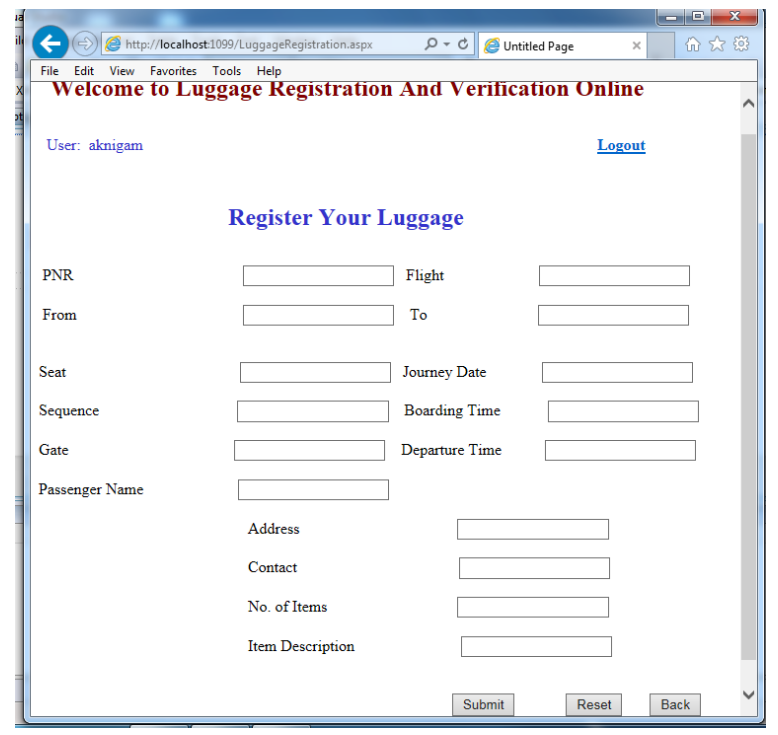

Fig-3: Luggage Registration Process

Fig-4 Shows the main page of user after login for luggage registration and verification.

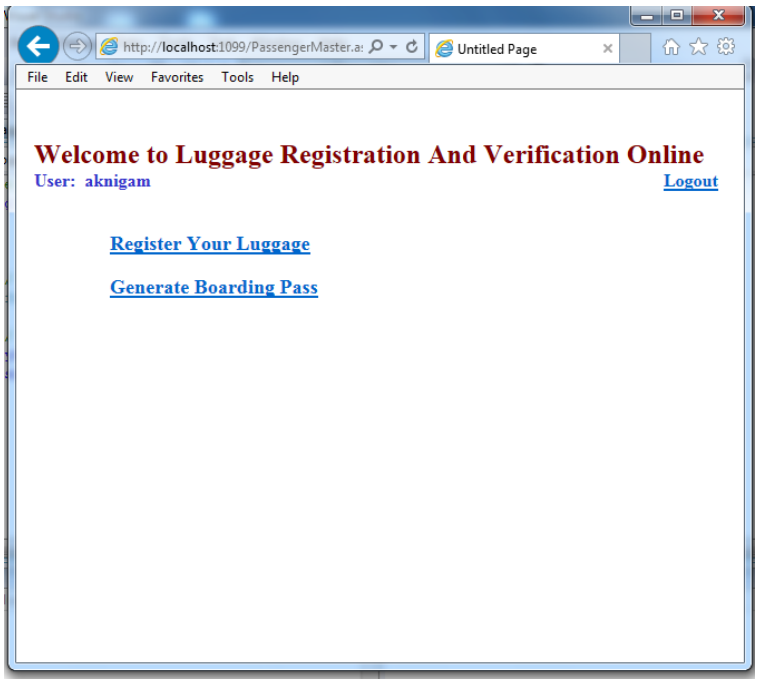

Fig-4: Main Page of User

Fig-5(a) shows the generation of boarding pass and QR code for luggage items.

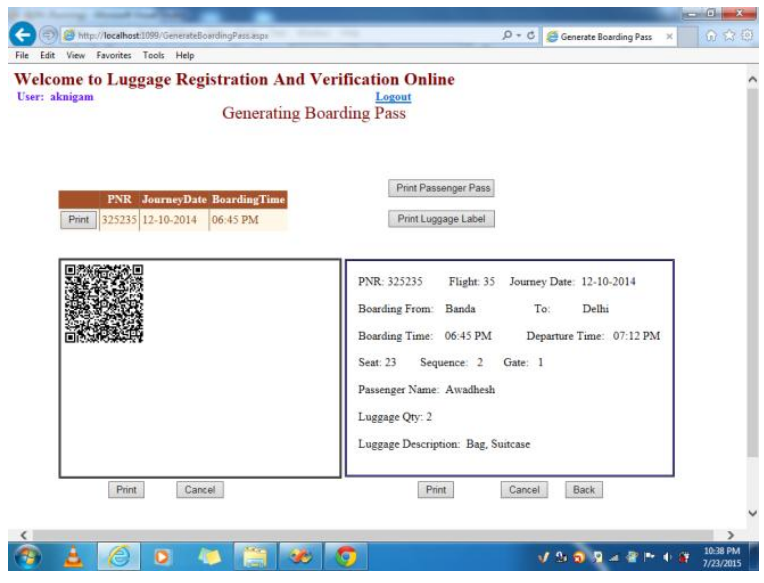

\section{Fig-5(a): Generation of Boarding Pass with QR code}

Fig-5(b) shows the successful verification of luggage information encoded in $\mathrm{QR}$ code. The QR code on luggage items is read by barcode scanner. Here we verifying the $Q R$ code by browsing the image of QR code.

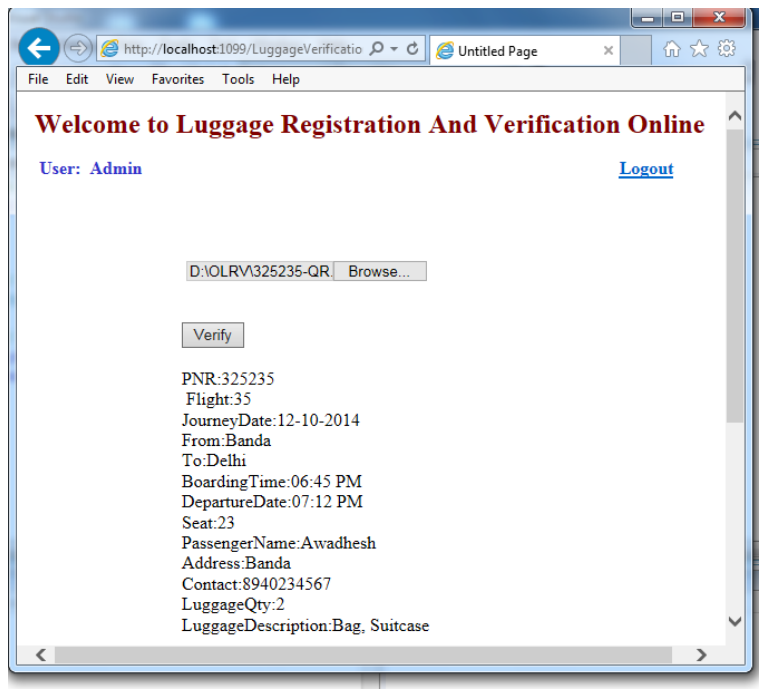

Fig-5(b): Verification of QR Code

\section{CONCLUSIONS}

Normal QR Codes can compress only up to $4 \mathrm{~KB}$ of data. Using the techniques followed here, the data capacity can be increased drastically. As compared to the normal QR Codes, the data capacity of the QR Code after following technique was found to be more than $4 \mathrm{~KB}$. Efficient data compression techniques can be used to store more than $4 \mathrm{~KB}$ of data inside a QR Code. A variety of data compression techniques can be used to obtain more data storage capacity. Comparing with the existing technologies used to generate bar codes, QR Codes were found to be of great advantage to the manufacturer because of its great data storage capacity, 
reading speed and accuracy. The data capacity was further improved by combining the most distinguishing features of compression and bar code generation. Using this novel technique of data compression followed by data encoding, the data storage capacity of QR Codes were increased drastically.

\section{FUTURE SCOPE}

Currently only Smartphone's are technically equipped to do this. Many users that have mobile phones that have cameras are unable to get $\mathrm{QR}$ reading software for their phones. Future enhancements focus on QR Encoding of images which is more than $4 \mathrm{~KB}$ of size. Secure QR Coding can also be implemented using encryption techniques. Also, more advanced data compression techniques can be used to add more to the data capacity of the normal QR Codes.

\section{REFERENCES}

[1] Xiaofei Feng, Herong Zheng, "Design and Realization of 2D Color Barcode with High Compression Ratio" 2010 International Conference On Computer Design And Appliations (ICCDA 2010), 978-1-4244-7164-51, 2010 IEEE, 978-1-4244-7164-51, 2010 IEEE, Volume 1

[2] Nancy Victor, "Enhancing the Data Capacity of QR Codes by Compressing the Data before Generation", International Journal of Computer Applications (0975- 8887), Volume 60 - No.2, December 2012.

[3] Peter Kieseberg, Manuel Leithner, Martin Mulazzani, Lindsay Munroe, Sebastian Schrittwieser, Mayank Sinha, Edgar WeipplT. J., "QR Code Security"

[4] Chun-lei XIA, "Examination Certificate Based on TwoDimensional Bar Code Technology", 2008 International Symposium on Computer Science and Computational Technology, 978-0-7695-3498-5/08/2008 IEEE DOI 10.1109/ISCSCT.2008.102

[5] Tasos Falas, Hossein Kashani, "Two-Dimensional Barcode Decoding with Camera-Equipped Mobile Phones", Proceedings of the Fifth Annual IEEE International Conference on Pervasive Computing and Communications Workshops(PerComW'07) 0-7695-2788-4/07/2007

[6] William Claycomb, Dongwan Shin, "Using A Two Dimensional Colorized Barcode Solution for Authentication in Pervasive Computing”, 1-4244-0237-9/06/2006 IEEE.

[7] M.Pitchaiah Philemon Daniel, Praveen, "Implemation of Advanced Encryption Standard Algorithm", nternational Journal of Scientific \& Engineering Research Volume 3, Issue 3, March -2012 1 ISSN 2229-5518.

[8] Guenther Starnberger, Lorenz Froihofer and Karl M. Goeschka, "QR-TAN: Secure Mobile Transaction Authentication", 2009 International Conference on Availability, Reliability and Security, 978-0-7695-35647/09 IEEE DOI 10.1109/ARES.2009.96
[9] ISO/IEC 18004:2000 Information Technology Automatic Identification and Data Capture Techniques Barcode Symbology- QR Code (MOD), June 2000.

[10] Sarah Lyons and Frank R. Kschischang, "TwoDimensional Barcodes for Mobile Phones", 25th Biennial Symposium on Communications, 978-1-4244-5711$3 / 10 / 2010$

[11] R. Bose and D. Ray-Chaudhuri. On a class of errorcorrecting binary group codes*. Information and control, 3(1):68\{79, 1960.

[12] David L. Donoho, Martin Vetterli, Fellow, IEEE, R. A. DeVore, and Ingrid Daubechies, Senior Member, IEEE," Data Compression and Harmonic Analysis", IEEE TRANSACTIONS ON INFORMATION THEORY, VOL. 44, NO. 6, OCTOBER 1998, 0018-9448/98\$10.00 ã 1998 IEEE

[13] Hee I1 Hahn and Joung Koo Joung, "Implementation of Algorithm to Decode Two-Dimensional Barcode PDF417”, ICSP'O2 Proceedings, 0-7803-7488-6/02/\$17.00 Q 2002 IEEE. 